\title{
Effect of Prenatal Betamethasone/Thyrotropin Releasing Hormone Treatment on Somatosensory Evoked Potentials in Preterm Newborns
}

\author{
FRANCIS DE ZEGHER, LINDA DE VRIES, VERONIQUE PIERRAT, HANS DANIELS, \\ BERNARD SPITZ, PAUL CASAER, HUGO DEVLIEGER, AND EPHREM EGGERMONT \\ Departments of Pediatrics and Obstetrics, University Hospital Gasthuisberg, 3000 Leuven, Belgium
}

\begin{abstract}
The maternal administration of betamethasone and thyrotropin releasing hormone (TRH) to accelerate the maturation of the fetus is an increasingly adopted strategy to prevent neonatal morbidity in preterm infants. The effect of this prenatal treatment on the neural maturation of the infant was assessed by measuring somatosensory evoked potentials (SEP) in preterm infants (gestational age 29-36 wk) on the 1st postnatal day, at the age of $1 \mathrm{wk}$, and before discharge. The N1 latency values of the SEP obtained in 14 infants who were exposed prenatally to betamethasone/TRH were compared with the N1 latencies measured in 12 control infants. On the 1st postnatal day, the N1 latencies in the betamethasone/ TRH-treated infants were strikingly shorter $(p<0.01)$ than in the controls. However, at the age of 1 wk and at discharge, the $\mathrm{N} 1$ latency values of both groups were similar. In conclusion, the present study provides the first solid evidence for the concept that the prenatal exposure to betamethasone/TRH accelerates the SEP-assessed neural maturation of the human fetus, that this prenatal acceleration is followed by a compensatory relative deceleration during the early neonatal period, and that the subsequent SEP-assessed neural maturation proceeds at a normal velocity. (Pediatr Res 32: 212-214, 1992)
\end{abstract}

Abbreviations

TRH, thyrotropin releasing hormone SEP, somatosensory evoked potential

Respiratory distress in preterm infants is an important cause of neonatal mortality and morbidity and is responsible for a significant part of long-term child disability. One of the strategies being developed to tackle this problem is to administer, in the case of threatening premature birth, a treatment to the fetus that accelerates fetal maturation, including the preparation for neonatal respiration. The most advanced of these treatment schedules consists of the combined administration of glucocorticoids and TRH to the pregnant woman, with both hormones reaching the fetus transplacentally (1-3). At present, this strategy is still considered by many obstetricians to be in an experimental stage and is therefore not routinely applied. As a consequence, some of the preterm newborns referred to our neonatal unit did receive this prenatal therapy, whereas others did not. This setting provided the opportunity to study the effect of prenatal treatment

Received November 7, 1991; accepted February 20, 1992

Correspondence and reprint requests: Francis de Zegher, Department of Pediatrics, University Hospital Gasthuisberg, 3000 Leuven, Belgium.

Supported by a grant from the Medical Research Council, Belgium (FGWO) and a grant from COMETT, Brussels, Belgium. with betamethasone/TRH on the neural maturation of preterm infants. SEP were used to assess the effect of prenatal betamethasone/TRH treatment on neural maturation because this technique is particularly suited for noninvasive evaluation of the integrity and functional maturation of several afferent pathways in the nervous system.

\section{SUBJECTS AND METHODS}

Twenty-six preterm infants, admitted to the neonatal unit, were enrolled in this prospective, controlled, longitudinal study. Informed parental counsel was obtained in each case. All infants had a gestational age of 36 wk or less. Gestational age was obtained from maternal dates, early prenatal ultrasound assessments when available, and clinical measurements according to the Ballard score (4). Infants were eligible for participation in the study when the following criteria were met: 1) no perinatal asphyxia (the 1-min Apgar score above 7); 2) less than 30\% oxygen requirement beyond the first $12 \mathrm{~h}$ of postnatal life; 3) normal cranial ultrasound scan on at least two occasions; 4) serum bilirubin level less than $250 \mu \mathrm{mol} / \mathrm{L} ; 5)$ normal results on sequential neurologic examination; 6) either no prenatal exposure to betamethasone/TRH (control group) or prenatal treatment with at least one standardized course of betamethasone (two doses of $12 \mathrm{mg}$ intramuscularly every $24 \mathrm{~h}$ ) and TRH (UCB, Brussels, Belgium) (four doses of $400 \mu \mathrm{g}$ i.v. every $8 \mathrm{~h}$ ). All treated infants were delivered within $5 \mathrm{~d}$ after a full maturation course. Five women received multiple courses (three to five) on a weekly basis; two fetuses were exposed to TRH without betamethasone. Prenatal tocolytic treatment with $\beta$-agonists was administered to all pregnant women in preterm labor. The study population ( $n=26$ infants) was evaluated in two subgroups, based on the presence $(n=14)$ or absence $(n=12)$ of prenatal exposure to betamethasone/TRH. The mean gestational age of both groups was $33 \mathrm{wk}$, with a range of $29-36 \mathrm{wk}$ in the control group and a range of 32-34 wk in the pretreated group. There were three multiple pregnancies in both groups.

SEP were recorded with a Nihon Kohden Neuropack II model 5100 (Nihon Kohden, Tokyo, Japan), as described previously (5). $\mathrm{Ag} / \mathrm{AgCl}$ disc electrodes were applied; the negative electrode on $\mathrm{C}^{\prime}$, the positive on $\mathrm{Fz}$, and the neural on the lower arm. C3' was marked with permanent ink. The impedance was usually between 2 and $5 \mathrm{kohm}$ and never more than $10 \mathrm{kohm}$. Electrical stimuli of $0.1 \mathrm{~ms}$ duration were delivered at the rate of 1 every $2 \mathrm{~s}$ with a hand-held device that was placed on the ventral part of the wrist, overlying the median nerve. The stimulation intensity used was that necessary to produce a minimal thumb twitch. A total of 128 responses were averaged through a bandpass of 2$100 \mathrm{~Hz}$. Each trial was repeated two or three times to ensure reproducibility. The analysis time was $100 \mathrm{~ms}$. The latency to the peak of N1 was measured according to standardized criteria 
(6). All infants were studied without sedation during natural sleep.

Sequential SEP were performed in all infants on $\mathrm{d} 1$ (within $24 \mathrm{~h}$ after birth) and d 8 of postnatal life and in 24 of 26 infants a third time, before discharge from the unit. These longitudinally obtained N1 latency values were interpreted in view of the available, cross-sectionally collected, normal range $(95 \%$ tolerance limits) measured in preterm infants after the immediate postnatal period, using identical filter settings, stimulation rate, and analysis time (7). We have previously demonstrated that the normal N1 latencies during the first postnatal week are longer and accelerate faster than the N1 latencies observed afterwards (5).

To compare the SEP results of the two groups statistically, the $\mathrm{N} 1$ latency values were transformed into $\mathrm{SD}$ scores according to the aforementioned N1 latency reference range. The MannWhitney U-test was used for statistical comparisons between groups.

\section{RESULTS}

The results of the sequential SEP, expressed in N1 latency values and related to the age of the infant, are represented in Figure 1. The control infants (upper panel) consistently developed the longitudinal N1 latency pattern of the "normal" low-
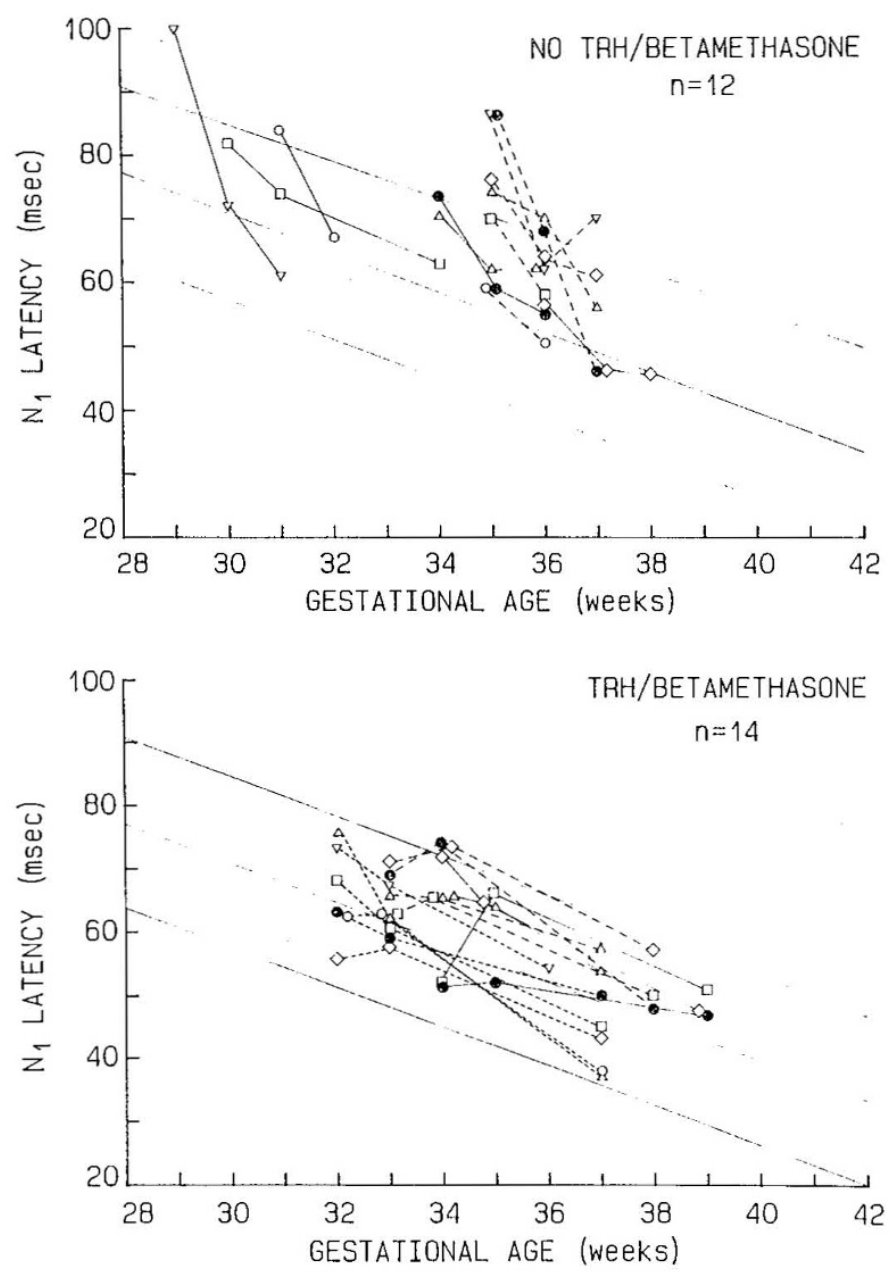

Fig. 1. Sequential SEP results, expressed as N1 latency values for postconceptional age, obtained in control preterm newborns (upper panel) and in preterm newborns treated prenatally with betamethasone/ TRH (lower panel). Identical, interconnected symbols identify the sequential results measured in each infant. The displayed reference range ( $95 \%$ tolerance limits) for newborns after the immediate postnatal period is derived from Ref. 7. risk preterm infant, as reported previously (5). The N1 latency values observed in these infants are strikingly elevated on the day of birth, being localized outside the reference range in eight of 12 infants. They decrease remarkably during the 1 st postnatal week and evolve subsequently within the limits of the crosssectionally obtained reference range.

In sharp contrast, infants who were treated prenatally with betamethasone/TRH (Fig. 1, lower panel) display a longitudinal N1 latency pattern with different characteristics. The N1 latency values observed in these infants on the day of birth are localized within the reference range in 14 of 14 infants. Furthermore, the decrease in N1 latencies during the 1st postnatal week is much less pronounced than in the control group. As a result, the difference in $\mathrm{N} 1$ latencies between the two groups at birth is no longer present by the end of the 1st postnatal week. Subsequently, the $\mathrm{N} 1$ latencies of prenatally treated infants also follow a course within the reference range.

The described visual impression was confirmed statistically by transforming the respective N1 latency values into SD scores that account for the effect of gestational age on the SEP results (see Subjects and Methods).

On d 1, the median N1 latency SD score of the control group was 2.1 , with the 25 th-75th percentile range extending from 1.6 to $3.4 \mathrm{SD}$; the median SD score of the pretreated group was 0.6 with a range from -0.5 to $1.5 \mathrm{SD}$. Statistically, the difference between the results of the two groups was highly significant $(p<$ $0.001)$. The difference of $1.5 \mathrm{SD}$ between the medians of the groups corresponds to a median maturational effect of $3.7 \mathrm{wk}$ by the prenatal betamethasone/TRH exposure.

On $\mathrm{d} 8$, there was no significant difference between the results of the two groups anymore, the median SD score in the nonpretreated group being 0.8 (range 0.3 to $1.6 \mathrm{SD}$ ) and 1.0 in the pretreated group (range -0.3 to $1.3 \mathrm{SD}$ ). The results of the SEP performed in both groups before discharge were also comparable, the median SD score being 0.8 (range -0.3 to $1.4 \mathrm{SD}$ ) in the nonpretreated group and 0.6 in the pretreated group (range -0.5 to $0.8 \mathrm{SD})$.

\section{DISCUSSION}

The neural maturation of the human infant, as assessed by SEP, appears to accelerate normally during the 1st postnatal week when compared to the prenatal maturation and to the maturation later in infancy. The evidence supporting this concept was previously collected from infants, born near term, who had not been exposed prenatally to glucocorticoids or TRH (5). The data obtained in the control infants of this study corroborate this principle and extend it to infants born as premature as $29 \mathrm{wk}$ of gestation.

The N1 latency of SEP observed within $24 \mathrm{~h}$ after birth in newborns exposed prenatally to betamethasone/TRH was strikingly shorter than the N1 latency documented in control newborns. In addition, the results of the SEP obtained in both groups at the end of the 1st neonatal week and before discharge appeared to be similar. These observations suggest that the SEP-assessed neural maturation of infants prenatally treated with betamethasone/TRH is relatively accelerated before birth and decelerates to a compensatory extent during the 1 st week after birth. As a result, both groups of infants appear to reach a comparable state of SEP-assessed neural maturation by the end of the 1st postnatal week.

The physiologic mechanisms underlying the observed difference in SEP results between the betamethasone/TRH-exposed and control infants are at present unclear. The difference appears to be independent of the respiratory condition of the infants, inasmuch as infants with major respiratory distress were excluded from the study. The actions of thyroid hormones and glucocorticoids are likely to be important determinants of neonatal SEP responses. First, the normal acceleration of the decrease in N1 latency within the first days after birth occurs in parallel with the 
physiologic activation of the thyroid axis (8). Moreover, the SEP responses measured in newborn infants with congenital primary hypothyroidism have been found to be delayed when compared to those in healthy controls (9). Furthermore, the prenatal administration of TRH to the pregnant woman has been shown to vigorously stimulate the fetal thyroid axis (10). Finally, glucocorticoids are known to be strikingly synergistic with thyroid hormones in advancing multiple aspects of fetal maturation, possibly in part by augmenting the peripheral conversion of tetraiodothyronine into the active triiodothyronine in the fetus $(1,8,11)$.

In spite of their striking significance, the present results should be interpreted with caution, in view of the design of the study. Although our evaluation was standardized, longitudinal, prospective, and controlled, the examined infants had not been strictly randomized for their prenatal treatment before referral to our neonatal unit. This suboptimal design was tolerated at the inception of this study because it was considered unacceptable to randomize the infants for a well-underbuilt, reportedly beneficial prenatal treatment $(1,3)$. In conclusion, the present study provides solid evidence supporting the concept that prenatal exposure to betamethasone/TRH accelerates the SEP-assessed neural maturation of the human fetus, that this prenatal acceleration is followed by a compensatory relative deceleration during the early neonatal period, and that the subsequent SEPassessed neural maturation proceeds at a normal velocity.

\section{REFERENCES}

1. Morales WJ, O'Brien WF, Angel JL, Knuppel RA, Sawai S 1989 Fetal lung maturation: the combined use of corticosteroids and thyrotropin-releasing hormone. Obstet Gynecol 73:11-16

2. Ballard PL 1989 Hormonal control of lung maturation. Clin Endocrinol Metab 3:723-753

3. de Zegher F, Spitz B, Devlieger H 1992 The basis for the prenatal treatment with TRH to prevent neonatal respiratory distress. Arch Dis Child 67:450454

4. Ballard JL, Novak KK, Driver MBA 1979 A simplified score for assessment of fetal maturation of newly born infants. J Pediatr 95:769-774

5. Pierrat V, de Vries L, Minami T, Casaer P 1990 Somatosensory evoked potentials and adaptation to extrauterine life: a longitudinal study. Brain Dev 12:376-379

6. Desmedt JE, Brunko E, Debecker J 1974 Maturation of the somotosensory evoked potentials in normal infants and children with special reference to the early N1 component. Electroencephalogr Clin Neurophysiol 40:43-58

7. Klimach V, Cooke RWI 1988 Maturation of the neonatal somatosensory evoked responses in preterm infants. Dev Med Child Neurol 30:208-214

8. Fisher DA, Polk DH 1989 Development of the thyroid. Clin Endocrinol Metab 3:627-657

9. Bongers-Schokking CJ, Colon EJ, Hoogland RA, de Groot CJ, Van den Brande JL 1991 Somatosensory evoked potentials in neonates with primary congenital hypothyroidism during the first week of therapy. Pediatr Res 30:34-39

10. Thorpe-Beeston JG, Nicolaides KH, Snijders RJM, Butler J, McGregor AM 1991 Fetal thyroid stimulating hormone response to maternal administration of thyrotropin releasing hormone. Am J Obstet Gynecol 164:1244-1245

11. Osathanondh R, Chopra IJ, Tulchinsky D 1978 Effects of dexamethasone on fetal and maternal thyroxine, triiodothyroxine, reverse triiodothyroxine and thyrotropin levels. J Clin Endocrinol Metab 47:1236-1239 